\title{
ORIGINAL
}

\section{DESIGUALDADES EN MORTALIDAD TOTAL Y POR CAUSA DE MUERTE SEGÚN EL NIVEL DE ESTUDIOS EN NAVARRA: HALLAZGOS DE UN ESTUDIO LONGITUDINAL 2001-2008}

\author{
Estrella Miqueléiz (1), Lourdes Lostao (1), Laura Reques (2), Juan M Santos (3,4), María E. Calle (3,4) \\ y Enrique Regidor $(3,4,5)$.
}

(1) Departamento de Sociología. Universidad Pública de Navarra. Pamplona.

(2) Escuela Nacional de Sanidad. Instituto de Salud Carlos III. Madrid.

(3) Departamento de Medicina Preventiva. Salud Pública e Historia de la Ciencia. Facultad de Medicina. Universidad Complutense de Madrid. Madrid.

(4) Instituto de Investigación Sanitaria del Hospital Clínico San Carlos (IdISSC). Madrid.

(5) CIBER Epidemiología y Salud Pública (CIBERESP). Madrid.

\section{RESUMEN}

Fundamentos: Dada la ausencia de evidencia científica, el objetivo fue mostrar las desigualdades en mortalidad según el nivel de estudios en Navarra y la contribución de las principales causas de defunción a la magnitud de desigualdades en la mortalidad por todas las causas de muerte.

Métodos: Todos los ciudadanos de 25 años y mayores residentes en $\mathrm{Na}$ varra en 2001 fueron seguidos durante 7 años para conocer su estado vital. El indicador de posición socioeconómica utilizado fue el nivel de estudios. Se estimaron las tasas de mortalidad general y por causa de muerte ajustadas por edad según la educación. Posteriormente, se calcularon la diferencia relativa (razón) y la diferencia absoluta de tasas entre las categorías más baja y más alta de nivel de estudios y la contribución de las principales causas de muerte a la diferencia absoluta.

Resultados: La razón de tasas por todas las causas de muerte fue 1,37 en hombres y 1,23 en mujeres. El virus de la inmunodeficiencia humana (VIH) $(25,84)$ y los accidentes no intencionales $(3,78)$ presentaron las razones de tasas más altas en los hombres y la diabetes mellitus $(4,92)$ y el VIH $(4,38)$ en las mujeres. Las enfermedades cardiovasculares constituyeron la causa de muerte que más contribuyó a la diferencia absoluta en mortalidad: $26 \%$ en hombres y $48 \%$ en mujeres.

Conclusiones: La tasa de mortalidad en la población navarra muestra un gradiente inverso con el nivel educativo, a excepción de algunas localizaciones de cáncer. Las enfermedades cardiovasculares son la causa de muerte que más contribuye a las desigualdades absolutas en mortalidad, mientras que otras causas de muerte que muestran importantes desigualdades relativas contribuyen poco a las desigualdades absolutas.

Palabras clave: Desigualdades en mortalidad. Causa de muerte. Nivel de estudios. Navarra

Correspondencia

Estrella Miqueléiz

Departamento de Sociología.

Universidad Pública de Navarra

Campus de Arrosadía s/n. 31006 Pamplona, Navarra.

emikeleiz@yahoo.es

\section{ABSTRACT \\ Inequalities in Total Mortality and by Cause of Death According to the Level of Education in Navarra: Findings from a Longitudinal Study from 2001 to 2008}

Background: Due to the lack of evidence, the objective was to show the inequalities in mortality by educational level in Navarra and the contribution of the main causes of death to the magnitude of inequalities in mortality from all causes of death.

Methods: All citizens aged 25 years and older residing in Spain in 2001 were followed during 7 years to determine their vital status. Level of education was used as socioeconomic status indicator. It was estimated the age-adjusted total mortality rate and mortality rate from cause-specific mortality by educational level. Then it was calculated the relative difference (ratio) and the absolute difference in rates between the lowest and highest levels of education and the contribution of the main causes of death to the absolute difference.

Results: The rate ratio for all causes of death was 1.37 in men and 1.23 in women. The human immunodeficiency virus (HIV) (25.84) and unintentional injuries (3.78) are the causes of death with higher rate ratio in men and diabetes (4.92) and HIV (4.38) in women. Cardiovascular diseases were the leading causes of death that contribute most to the absolute difference in mortality: $26 \%$ in men and $48 \%$ women.

Conclusions: The mortality rate in the Navarre population shows an inverse gradient with educational level, except in some cancer sites. Cardiovascular disease is the leading cause of death that contributes most to the absolute inequalities in mortality, while other causes of death that show significant relative inequalities, contribute little to the absolute inequalities.

Keywords: Inequalities in mortality. Cause of death. Educational level. Navarra.

DOI: 


\section{INTRODUCCIÓN}

Todas las comunidades autónomas iniciaron alrededor del año 1990 sus primeros planes de salud. Algunos autores analizaron en el año 2005 la sensibilidad de estos planes a las desigualdades socioeconómicas en salud. La conclusión de aquel análisis fue la escasa atención que los planes de salud prestaban a este asunto, con excepción del País Vasco ${ }^{1}$.

Navarra era una de las comunidades que presentaban índices de sensibilidad muy bajos, tanto en la descripción de la situación de salud según la posición socioeconómica, como en las propuestas de acción concretas en grupos socioeconómicos de población, en los que las intervenciones pueden ser prioritarias a fin de reducir determinados problemas de salud. Sin embargo, en el Plan de Salud de Navarra de $2014-2020^{2}$ ya se incluyen las desigualdades socioeconómicas en salud en la agenda política y, además, se menciona explícitamente este asunto como una de las líneas de investigación prioritarias debido a la limitada evidencia empírica.

Con el propósito de reducir la escasa evidencia sobre las desigualdades en salud en Navarra, el objetivo de este estudio fue analizar las desigualdades en mortalidad según el nivel de estudios en Navarra y la contribución de las principales causas de defunción a la magnitud de desigualdades en la mortalidad por todas las causas de muerte.

\section{MATERIAL Y MÉTODO}

La fuente de datos fue la población del censo de 2001 a la que se siguió durante siete años y dos meses para conocer su estado vital. Los datos fueron elaborados por el Instituto Nacional de Estadística en base a los registros individuales del censo cruzados con el registro de población y con el registro de mortalidad mediante el uso de identificadores comunes. Los fallecimientos se refieren a las personas que murieron entre el 1 de noviembre de 2001 (fecha de realización del censo) y el 31 de diciembre de 2008 (fecha de finalización del periodo de seguimiento). El Instituto Nacional de Estadística proporcionó a los investigadores el fichero final con los datos, después de eliminar las variables que pudieran tener gran capacidad identificativa.

La cohorte final de seguimiento quedó constituida por 40.148.305 individuos, después de excluir al $1,7 \%$ de los sujetos que no pudieron ser encontrados en el padrón continuo ni en el registro de mortalidad durante el seguimiento. Para el presente estudio solo se seleccionaron los 549.800 individuos residentes en la comunidad autónoma de Navarra.

El indicador de posición socioeconómica utilizado fue el nivel de estudios. Esta variable se recogió en el censo y se refiere al nivel de estudios más alto completado por cada individuo. Para el presente estudio esta variable se agrupó en las siguientes cuatro categorías a partir de las diez que aparecían en el cuestionario: personas con estudios de primer grado o inferiores, personas que completaron el primer ciclo del segundo grado, personas que completaron el segundo ciclo del segundo grado y personas con estudios universitarios. Se incluyó en el análisis a los 407.443 sujetos con edad mayor o igual a 25 años a fecha de 1 de noviembre de 2001 , ya que fueron los que potencialmente podían haber obtenido el mayor grado de escolarización, Después de excluir a 7.128 $(1,3 \%)$ sujetos por ausencia de información sobre el nivel de estudios, se analizaron 3.750.530,21 personas-año en riesgo y 32.011 defunciones.

Análisis estadístico. Los análisis se realizaron por separado en mujeres y en hombres. En primer lugar, se analizó al conjunto de la población de cada sexo y posteriormente por separado cuatro grupos de edad: 25 a 44 años, 45 a 64 años, 65 a 74 años y 75 años y más. Se calcularon las tasas de mortalidad ajustadas por edad en cada categoría de educación tomando como referencia para el ajuste la población estándar europea. Se esti- 
mó la tasa de mortalidad general y la tasa de mortalidad por las causas de muerte que producen mayor número de fallecimientos. Las causas de muerte analizadas fueron cáncer, enfermedades cardiovasculares, enfermedades respiratorias, enfermedades digestivas, diabetes mellitus, septicemia, sida, accidentes no intencionales y suicidio. Estas causas de muerte representaron el $80 \%$ de las defunciones en la población analizada. En los grupos de edad se mostraron únicamente los hallazgos para las cuatro causas de muerte más prevalentes en cada grupo.

A continuación se estimaron las diferencias relativas (razones de tasas) y absolutas (diferencias de tasas) entre la categoría más baja de nivel de educación y la categoría más alta. Los intervalos de confianza de las razones y diferencia de tasas se calcularon a partir de la varianza estimada mediante el análisis estratificado ${ }^{3}$. Asimismo, se calculó la contribución de cada causa de muerte a las diferencias absolutas en mortalidad, con el porcentaje que representó cada causa de muerte con respecto a la diferencia absoluta en la mortalidad total.

\section{RESULTADOS}

La tabla 1 muestra el número de personasaño en riesgo y el número de muertes según el nivel de estudios en mujeres y en hombres. El número de personas-año en riesgo y el número de muertes fue mayor en el grupo de menor nivel de estudios, tanto en hombres como en mujeres (10.794 muertes en hombres con educación primaria o menos frente a 973 en el grupo de estudios universitarios). En el grupo de 25 a 44 años el número de personas-año en riesgo fue mayor en el grupo de estudios universitarios.

Las tablas 2 y 3 presentan en hombres y mujeres, respectivamente, las tasas de mortalidad estandarizadas por edad, según la causa de muerte en cada categoría de nivel de estudios, la razón y la diferencia de tasas entre la categoría más baja y más alta del nivel educativo y la contribución de cada causa de muerte a la diferencia en mortalidad por todas las causas. La razón de tasas de mortalidad por todas las causas de muerte fue 1,37 en hombres y 1,23 en mujeres. Entre las grandes causas de muerte, la razón de tasas de mortalidad más alta en los hombres se observó en el VIH $(25,84)$, los accidentes no intencionales $(3,78)$ y las enfermedades del aparato digestivo $(3,36)$ y en las mujeres en la diabetes mellitus $(4,92)$, el VIH $(4,38)$ y las enfermedades del aparato respiratorio $(1,87)$. En mujeres, la razón de tasas de mortalidad por cáncer fue menor que $1(0,92)$, es decir, la tasa fue mayor en las que presentaron un mayor nivel de estudios.

En esas tablas también se observa que la diferencia de la tasa de mortalidad por estas cuatro causas de muerte fue 392,0 y 142,1 por 100.000 personas-año en hombres y mujeres, respectivamente. En hombres, la diferencia absoluta entre las tasas de mortalidad en las categorías más baja y más alta de nivel de estudios fue $26,2 \%$ y se debió a las enfermedades cardiovasculares (tabla 3). La contribución del cáncer, las enfermedades digestivas y los accidentes fue del 19,5\%,14,0\%, y $12,4 \%$ respectivamente. El $48,1 \%$ de la diferencia absoluta entre las tasas de mortalidad en las categorías más baja y más alta de nivel de estudios en mujeres se debió a las enfermedades cardiovasculares (tabla 2). La contribución de las enfermedades respiratorias y la diabetes mellitus fue del 25,6\% y $13,4 \%$, respectivamente. La contribución de la mortalidad por cáncer fue negativa, puesto que la tasa de mortalidad fue mayor en las mujeres con mayor nivel de estudios.

La tabla 4 muestra en hombres los mismos cálculos que la tabla 2 pero en diferentes grupos de edad y para las cuatro principales causas de muerte. Las causas de muerte que tuvieron la razón de tasas más alta fueron el VIH en el grupo de 25 a 44 años (dato no mostrado) y las enfermedades digestivas en el resto de los grupos de edad (4,70 en el grupo de 45 a 64 años). Los accidentes fueron la causa de muerte que más contribuyó 


\begin{tabular}{|c|c|c|c|c|}
\hline \multicolumn{5}{|c|}{$\begin{array}{c}\text { Tabla } 1 \\
\text { Número de personas-año a riesgo y número de muertes por grupo de edad nivel } \\
\text { de estudios y sexo en la población de } 25 \text { años de edad y más. Navarra 2001-2008 }\end{array}$} \\
\hline \multirow[b]{2}{*}{ Edad y nivel de estudios } & \multicolumn{2}{|c|}{ Hombres } & \multicolumn{2}{|c|}{ Mujeres } \\
\hline & $\begin{array}{c}\text { Personas-año } \\
\text { en riesgo }\end{array}$ & $\begin{array}{c}\text { Número de } \\
\text { muertes }\end{array}$ & $\begin{array}{l}\text { Personas-año } \\
\text { en riesgo }\end{array}$ & $\begin{array}{c}\text { Número de } \\
\text { muertes }\end{array}$ \\
\hline \multicolumn{5}{|l|}{ Total } \\
\hline Educación primaria o menos & $697.755,68$ & 10.794 & $745.699,80$ & 10.847 \\
\hline Educación secundaria de $1^{\circ}$ ciclo & $500.145,22$ & 4.024 & $484.712,73$ & 2.841 \\
\hline Educación secundaria de $2^{\circ}$ ciclo & $427.294,77$ & 1.425 & $359.692,46$ & 602 \\
\hline Estudios universitarios & $239.765,24$ & 973 & $295.464,31$ & 505 \\
\hline \multicolumn{5}{|l|}{25 a 44} \\
\hline Educación primaria o menos & $81.176,76$ & 196 & $60.397,74$ & 75 \\
\hline Educación secundaria de $1^{\circ}$ ciclo & $200.641,31$ & 342 & $165.151,93$ & 144 \\
\hline Educación secundaria de $2^{\circ}$ ciclo & $222.473,96$ & 230 & $188.830,87$ & 113 \\
\hline Estudios universitarios & $144.088,45$ & 82 & $197.183,48$ & 72 \\
\hline \multicolumn{5}{|l|}{45 a 64} \\
\hline Educación primaria o menos & $137.139,54$ & 1.284 & $153.741,23$ & 569 \\
\hline Educación secundaria de $1^{\circ}$ ciclo & $161.249,31$ & 1.105 & $182.293,96$ & 443 \\
\hline Educación secundaria de $2^{\circ}$ ciclo & $85.757,34$ & 486 & $55.223,41$ & 137 \\
\hline Estudios universitarios & $61.101,78$ & 276 & $51.624,70$ & 128 \\
\hline \multicolumn{5}{|l|}{65 a 74} \\
\hline Educación primaria o menos & $98.549,25$ & 2.926 & $124.778,02$ & 1.683 \\
\hline Educación secundaria de $1^{\circ}$ ciclo & $40.861,88$ & 1.038 & $48.243,17$ & 533 \\
\hline Educación secundaria de $2^{\circ}$ ciclo & $10.634,45$ & 279 & $8.425,28$ & 79 \\
\hline Estudios universitarios & $10.789,91$ & 248 & $7.129,20$ & 75 \\
\hline \multicolumn{5}{|l|}{75 y más } \\
\hline Educación primaria o menos & $64.016,71$ & 6.388 & $117.475,59$ & 8.520 \\
\hline Educación secundaria de $1^{\circ}$ ciclo & $17.093,70$ & 1.539 & $28.646,19$ & 1.721 \\
\hline Educación secundaria de $2^{\circ}$ ciclo & $4.189,50$ & 430 & $4.716,95$ & 273 \\
\hline Estudios universitarios & $4.627,87$ & 367 & $3.878,91$ & 230 \\
\hline
\end{tabular}

a las diferencias absolutas en mortalidad en los hombres de 25-44 años de edad, el cáncer en hombres de 45 a 74 años y las enfermedades cardiovasculares en los hombres de 75 años y más.

La tabla 5 muestra en mujeres los mismos cálculos que la tabla 3, pero en diferentes grupos de edad y para las cuatro principales causas de muerte en cada grupo. Para todas las causas de mortalidad, la tasa de mortalidad fue mayor en las mujeres con nivel educativo más bajo (2,72 en mujeres de 25 a 44 años), a excepción de la mortalidad por cáncer en las mujeres mayores de 45 años $(0,87$ en el grupo de 45 a 64 años). Las principales causas de muerte que tuvieron la razón de tasas más alta fueron el VIH en el grupo de 25 a 44 años y las enfermedades cardiovasculares en el resto de grupos de edad (dato no mostrado). El cáncer fue la causa de muerte que más contribuyó a las diferencias absolutas en la mortalidad entre la categoría más baja y más alta de nivel de estudios en el grupo de 25-44 años de edad (15,9\%). Por encima de los 44 años la causa de la muerte que contribuyó en mayor proporción fueron las enfermedades cardiovasculares. 


\section{Tabla 2}

Tasa de mortalidad estandarizada por edad por $\mathbf{1 0 0 . 0 0 0}$ personas-año a riesgo por causa de muerte según el nivel educativo, razón de tasas de mortalidad (RT), diferencia de tasas de mortalidad por $\mathbf{1 0 0 . 0 0 0}$ personas-año (DT) e intervalos de confianza al $95 \%$ (IC95\%) de la razón y la diferencia. Cohorte de hombres de 25 años de edad y más en Navarra 2001 a 2008

\begin{tabular}{|c|c|c|c|c|c|c|c|c|c|c|}
\hline $\begin{array}{l}\text { Causa de muerte } \\
\text { (código CIE-10") }\end{array}$ & Total & $\begin{array}{c}\text { Educación } \\
\text { primaria } \\
\text { o menos }\end{array}$ & $\begin{array}{l}\text { Educación } \\
\text { secundaria } \\
\text { de } 1^{\circ} \text { ciclo }\end{array}$ & $\begin{array}{l}\text { Educación } \\
\text { secundaria } \\
\text { de } 2^{\circ} \text { ciclo }\end{array}$ & $\begin{array}{c}\text { Estudios } \\
\text { universitarios }\end{array}$ & $\mathbf{R T}^{1}$ & IC $95 \%$ & $\mathrm{DT}^{2}$ & IC $95 \%$ & $\begin{array}{c}\text { Contribución } \\
\text { a la diferencia en la } \\
\text { mortalidad por todas } \\
\text { las causas }(\%)^{3} \\
\end{array}$ \\
\hline Todas las causas & $1.256,9$ & $1.461,7$ & $1.317,0$ & $1.314,2$ & $1.069,7$ & 1,37 & $1,33 \mid 1,40$ & 392,0 & $356,7 \mid 427,3$ & 100,0 \\
\hline Cáncer (C00-C99) & 420,2 & 441,7 & 432,4 & 450,8 & 365,0 & 1,21 & $1,15 \mid 1,27$ & 76,6 & $56,0 \mid 97,3$ & 19,5 \\
\hline Pulmón (C32) & 110,4 & 111,3 & 110,5 & 127,1 & 93,1 & 1,19 & $1,08 \mid 1,33$ & 18,1 & $7,2 \mid 29,0$ & 4,6 \\
\hline Próstata (C61) & 40,2 & 40,6 & 42,9 & 43,0 & 32,7 & 1,24 & $1,10 \mid 1,40$ & 7,9 & \begin{tabular}{l|l}
3,1 & 12,6
\end{tabular} & 2,0 \\
\hline Colon (C18) & 35,7 & 37,7 & 34,4 & 32,1 & 41,9 & 0,90 & $0,77 \mid 1,05$ & $-4,2$ & $-10,2 \mid 1,8$ & $-1,1$ \\
\hline Vejiga (C67) & 24,5 & 25,6 & 24,8 & 20,8 & 23,8 & 1,08 & $0,91 \mid 1,27$ & 1,8 & $-2,4 \mid 6,1$ & 0,5 \\
\hline Estómago (C16) & 27,0 & 30,5 & 28,9 & 30,5 & 27,0 & 1,87 & $1,52 \mid 2,30$ & 14,2 & $\begin{array}{l}9,0 \mid 19,4 \\
\end{array}$ & 3,6 \\
\hline Páncreas (C25) & 21,7 & 23,4 & 23,5 & 23,5 & 14,8 & 1,58 & \begin{tabular}{l|l}
$2,25 \mid 2,00$ \\
\end{tabular} & 8,7 & \begin{tabular}{l|l}
3,9 & 13,4
\end{tabular} & 2,2 \\
\hline Recto (C20) & 13,5 & 12,7 & 18,0 & 13,0 & 13,3 & 0,95 & $0,73 \mid 1,24$ & $-0,6$ & $-4,0 \mid 2,7$ & $-0,2$ \\
\hline Esófago (C15) & 12,5 & 14,3 & 12,1 & 12,9 & 13,8 & 1,04 & $0,78 \mid 1,37$ & 0,5 & $-3,5 \mid 4,4$ & 0,1 \\
\hline Laringe $(\mathrm{C} 32)$ & 12,2 & 19,5 & 10,2 & 11,0 & 9,4 & 2,07 & $1,59 \mid 2,71$ & 10,1 & $5,4 \mid 14,7$ & 2,6 \\
\hline Enfermedades cardiovasculares (I00-I99) & 379,1 & 401,5 & 369,9 & 395,4 & 298,7 & 1,34 & $1,28 \mid 1,40$ & 102,8 & \begin{tabular}{l|l|l}
85,5 & 120,1
\end{tabular} & 26,2 \\
\hline Cerebrovasculares 8(I60-I69) & 140,8 & 148,7 & 140,7 & 156,4 & 112,0 & 1,33 & $1,22 \mid 1,44$ & 36,7 & $25,4 \mid 48,0$ & 9,4 \\
\hline Isquémicas (I20-I25) & 88,9 & 92,8 & 83,5 & 103,1 & 58,5 & 1,59 & $1,46 \mid 1,73$ & 34,3 & $26,9 \mid 41,7$ & 8,8 \\
\hline Respiratorias (J00-J99) & 169,9 & 189,6 & 145,0 & 118,5 & 142,8 & 1,33 & $1,25 \mid 1,41$ & 46,8 & $36,2 \mid 57,4$ & 11,9 \\
\hline Neumococo/influenza (J09-J19) & 74,1 & 86,2 & 64,9 & 34,1 & 51,6 & 1,67 & $1,54 \mid 1,81$ & 34,6 & $27,7 \mid 41,5$ & 8,8 \\
\hline $\mathrm{EPOC}^{* *}(\mathrm{~J} 44)$ & 39,7 & 44,0 & 32,7 & 45,7 & 23,6 & 1,86 & $1,63 \mid 2,12$ & 20,3 & $15,1 \mid 25,6$ & 5,2 \\
\hline Enfermedades digestivas (K00-K93) & 64,8 & 78,2 & 62,9 & 52,8 & 23,3 & 3,36 & $2,94 \mid 3,84$ & 54,9 & $47,0 \mid 2,8$ & 14,0 \\
\hline Cirrosis (K74) & 17,2 & 22,9 & 17,3 & 22,2 & 5,3 & 4,36 & $3,06 \mid 6,22$ & 17,7 & $12,6 \mid 22,8$ & 4,5 \\
\hline Diabetes mellitus (E10-E14) & 25,8 & 28,5 & 25,8 & 14,6 & 20,5 & 1,39 & $1,17 \mid 1,64$ & 8,0 & $3,4 \mid 12,5$ & 2,0 \\
\hline Accidentes (W00-X59) & 48,4 & 66,3 & 56,7 & 45,8 & $\mathbf{1 7 , 5}$ & 3,78 & $3,05 \mid 4,68$ & 48,7 & $\mathbf{3 8 , 9} \mid \mathbf{5 8 , 5}$ & 12,4 \\
\hline Tráfico (V00-V98) & 16,3 & 23,9 & 17,9 & 16,4 & 4,7 & 5,09 & $3,22 \mid 8,05$ & 19,2 & \begin{tabular}{l|l|l}
13,0 & 25,4
\end{tabular} & 4,9 \\
\hline Suicidio (X60-X84) & 14,7 & 15,9 & 16,8 & 10,3 & 9,3 & 1,71 & $1,11 \mid 2,64$ & 6,6 & \begin{tabular}{l|l|}
1,2 & 12,0 \\
\end{tabular} & 1,7 \\
\hline VHI (B20-B24) & 4,5 & 9,9 & 6,8 & 1,9 & 0,4 & 25,84 & $3,48 \mid 91,95$ & 9,5 & \begin{tabular}{l|l|l|}
5,3 & 13,8
\end{tabular} & 2,4 \\
\hline
\end{tabular}

${ }^{1} \mathrm{RT}=$ Razón entre la tasas de mortalidad en el grupo con educación primaria o menos y la tasa de mortalidad en el grupo de estudios universitarios. ${ }^{2}$ DT $=$ Diferencia entre la tasas de mortalidad en el grupo con educación primaria o menos y la tasa de mortalidad en el grupo de estudios universitarios. ${ }^{3}$ Representa la diferencia de tasas en esa causa de muerte con respecto a la diferencia en la tasa de mortalidad por todas las causas * CIE-10: Clasificación Internacional de Enfermedades, 10 ${ }^{\mathrm{a}}$ revisión ${ }^{* * *}$ EPOC: Enfermedad pulmonar obstructiva crónica 


\section{Tabla 3}

Tasa de mortalidad estandarizada por edad por 100.000 personas-año a riesgo por causa de muerte según el nivel educativo, razón de tasas de mortalidad (RT), diferencia de tasas de mortalidad por $\mathbf{1 0 0 . 0 0 0}$ personas-año (DT) e intervalos de confianza. Cohorte de mujeres de 25 años de edad y más en Navarra 2001 a 2008

\begin{tabular}{|c|c|c|c|c|c|c|c|c|c|c|}
\hline $\begin{array}{l}\text { Causa de muerte } \\
\text { (código CIE-10") }\end{array}$ & Total & $\begin{array}{c}\text { Educación } \\
\text { primaria } \\
\text { o menos }\end{array}$ & $\begin{array}{l}\text { Educación } \\
\text { secundaria } \\
\text { de } 1^{\circ} \text { ciclo }\end{array}$ & $\begin{array}{l}\text { Educación } \\
\text { secundaria } \\
\text { de } 2^{\circ} \text { ciclo }\end{array}$ & $\begin{array}{c}\text { Estudios } \\
\text { universitarios }\end{array}$ & $\mathbf{R T}^{1}$ & IC $95 \%$ & $\mathbf{D T}^{2}$ & IC $95 \%$ & $\begin{array}{c}\text { Contribución } \\
\text { a la diferencia } \\
\text { en la mortalidad } \\
\text { por todas las causas }(\%)^{3}\end{array}$ \\
\hline Todas las causas & 714,8 & 766,5 & 660,5 & 629,8 & 624,4 & 1,23 & $1,19|1,27|$ & 142,1 & $119,3 \mid 64,8$ & 100,0 \\
\hline Enfermedades cardiovasculares (I00-I99) & 225,2 & 242,4 & 203,3 & 190,0 & 174,1 & 1,39 & $1,34 \mid 1,45$ & 68,3 & $58,7 \mid 77,9$ & 48,1 \\
\hline Cerebrovasculares 8(I60-I69) & 66,8 & 74,0 & 59,4 & 46,3 & 42,7 & 1,73 & $1,60 \mid 1,88$ & 31,3 & $25,8 \mid 36,8$ & 22,0 \\
\hline Isquémicas (I20-I25) & 32,6 & 35,0 & 28,6 & 26,4 & 22,6 & 1,55 & $1,36 \mid 1,76$ & 12,4 & $0,8 \mid 16,4$ & 8,7 \\
\hline Cáncer (C00-C99) & 189,6 & 191,7 & 187,4 & 202,4 & 207,9 & 0,92 & $0,86 \mid 0,99$ & $-16,2$ & \begin{tabular}{l|l|}
$-30,8$ & $-1,7$ \\
\end{tabular} & $-11,4$ \\
\hline Mama (C50) & 29,6 & 28,9 & 30,3 & 34,0 & 33,4 & 0,87 & $0,70 \mid 1,08$ & $-4,4$ & $-0,1 \mid 2,2$ & $-3,1$ \\
\hline Colon (C18) & 20,2 & 20,0 & 19,7 & 23,6 & 23,6 & 0,84 & $0,69 \mid 1,03$ & $-3,7$ & $-0,8 \mid 5,7$ & $-2,6$ \\
\hline Pulmón (C32) & 16,7 & 16,5 & 14,0 & 28,6 & 20,0 & 0,82 & $0,61 \mid 1,11$ & $-3,6$ & $-0,9 \mid 1,7$ & $-2,5$ \\
\hline Páncreas (C25) & 14,1 & 14,6 & 13,8 & 14,4 & 19,4 & 0,75 & $0,59 \mid 0,96$ & $-4,8$ & $-8,7 \mid-0,8$ & $-3,4$ \\
\hline Ovario (C56) & 11,4 & 11,2 & 12,1 & 7,6 & 14,4 & 0,77 & $0,58 \mid 1,03$ & $-3,3$ & $-6,8 \mid 2,3$ & $-2,3$ \\
\hline Estómago (C16) & 10,3 & 10,0 & 11,3 & 7,3 & 6,4 & 1,58 & $1,06 \mid 2,34$ & 3,7 & $0,6 \mid 6,7$ & 2,6 \\
\hline Linfoma (C81-C85) & 5,6 & 5,6 & 6,1 & 4,8 & 3,8 & 1,46 & $1,00 \mid 2,13$ & 1,8 & $-0,2|3,7|$ & 1,3 \\
\hline Leucemia (C91-C95) & 5,1 & 6,1 & 4,6 & 5,3 & 6,4 & 0,95 & $0,66 \mid 1,36$ & $-0,3$ & $-2,5 \mid 1,9$ & $-0,2$ \\
\hline Útero (C54) & 4,3 & 4,4 & 4,7 & 0,0 & 9,0 & 0,49 & $0,33 \mid 0,72$ & $-4,6$ & $-6,7 \mid-2,5$ & $-3,2$ \\
\hline Respiratorios (J00-J99) & 71,6 & 78,1 & 58,9 & 52,6 & 41,7 & 1,87 & $1,73 \mid 2,03$ & 36,4 & $31,1 \mid 41,7$ & 25,6 \\
\hline Neumococo/influenza (J09-J19) & 20,2 & 21,7 & 18,7 & 17,6 & 11,5 & 1,89 & $1,64 \mid 2,18$ & 10,2 & $7,4 \mid 12,9$ & 7,2 \\
\hline $\operatorname{EPOC}^{* *}(\mathrm{~J} 44)$ & 13,3 & 15,4 & 8,6 & 9,8 & 6,5 & 2,36 & $2,01 \mid 2,79$ & 8,9 & $6,5 \mid 11,2$ & 6,3 \\
\hline Enfermedades digestivas (K00-K93) & 33,8 & 36,8 & 29,3 & 30,2 & 27,9 & 1,32 & $1,17 \mid 1,48$ & 8,9 & $4,6 \mid 13,1$ & 6,3 \\
\hline Cirrosis (K74) & 4,2 & 6,1 & 3,1 & 0,7 & 8,1 & 0,75 & $0,48 \mid 1,18$ & $-2,0$ & $-4,8 \mid 0,8$ & $-1,4$ \\
\hline Diabetes mellitus (E10-E14) & 19,3 & 23,9 & 17,9 & 6,1 & 4,9 & 4,92 & $4,16|5,81|$ & 19,0 & $15,1 \mid 22,9$ & 13,4 \\
\hline Accidentes (W00-X59) & 18,6 & 19,6 & 18,2 & 19,2 & 18,9 & 1,04 & \begin{tabular}{l|l|}
0,81 & 1,33 \\
\end{tabular} & 0,7 & $-4,1 \mid 5,5$ & 0,5 \\
\hline Tráfico (V00-V98) & 6,4 & 5,1 & 6,7 & 5,6 & 4,5 & 1,13 & $0,58 \mid 2,18$ & 0,6 & $-2,5 \mid 3,6$ & 0,4 \\
\hline Suicidio (X60-X84) & 5,1 & 5,6 & 4,3 & 7,9 & 5,4 & 1,04 & $0,48 \mid 2,24$ & 0,2 & $-4,1 \mid 4,5$ & 0,1 \\
\hline VIH (B20-B24) & 1,8 & 6,6 & 2,5 & 0,8 & 1,5 & 4,38 & $2,28 \mid 8,43$ & 5,1 & $1,0 \mid 9,2$ & 3,6 \\
\hline
\end{tabular}

$\mathrm{RT}=$ Razón entre la tasas de mortalidad en el grupo con educación primaria o menos y la tasa de mortalidad en el grupo de estudios universitarios. ${ }^{2} \mathrm{DT}=\mathrm{Diferencia}$ entre la tasas de mortalidad en el grupo con educación primaria o menos y la tasa de mortalidad en el grupo de estudios universitarios. ${ }^{3}$ Representa la diferencia de tasas en esa causa de muerte con respecto a la diferencia en la tasa de mortalidad por todas las causas ${ }^{*} \mathrm{CIE}-10$ : Clasificación Internacional de Enfermedades, $10^{\mathrm{a}}$ revisión ${ }^{* *}$ EPOC: Enfermedad pulmonar obstructiva crónica 


\section{Tabla 4}

Tasa de mortalidad estandarizada por edad por 100.000 personas-año a riesgo por las principales causas de muerte según el nivel educativo, razón de tasas de mortalidad (RT), diferencia de tasas de mortalidad por 100.000 personas-año (DT) e intervalos de confianza al 95\% (IC95\%) de la razón y la diferencia.

Cohorte de hombres de diferentes grupos de edad en Navarra, 2001 a 2008

\begin{tabular}{|c|c|c|c|c|c|c|c|c|c|c|}
\hline Grupo de edad y causa de muerte & Total & $\begin{array}{l}\text { Educación } \\
\text { primaria } \\
\text { o menos }\end{array}$ & $\begin{array}{c}\text { Educación } \\
\text { secundaria } \\
\text { de } 1^{\circ} \text { ciclo }\end{array}$ & $\begin{array}{l}\text { Educación } \\
\text { secundaria } \\
\text { de } 2^{\circ} \text { ciclo }\end{array}$ & $\begin{array}{c}\text { Estudios } \\
\text { universitarios }\end{array}$ & $\mathbf{R T}^{1}$ & IC $95 \%$ & DT $^{2}$ & IC $95 \%$ & $\begin{array}{c}\text { Contribución } \\
\text { a la diferencia en la } \\
\text { mortalidad por todas } \\
\text { las causas }(\%)^{3}\end{array}$ \\
\hline \multicolumn{11}{|l|}{25 a 44} \\
\hline Todas las causas & 133,9 & 228,2 & 168,5 & 109,3 & 55,2 & 4,13 & $3,10 \mid 5,51$ & 173,0 & $137,9 \mid 208,2$ & 100,0 \\
\hline Cáncer (C00-C99) & 35,6 & 49,3 & 36,5 & 36,7 & 23,0 & 2,26 & $1,38 \mid 3,68$ & 27,4 & \begin{tabular}{l|l|l|}
10,6 & 44,3 \\
\end{tabular} & 15,8 \\
\hline Accidentes no intencionales (W00-X59) & 31,7 & 55,8 & 47,3 & 24,0 & 7,4 & 7,51 & $3,85 \mid 14,66$ & 48,4 & $31,2 \mid 65,6$ & 28,0 \\
\hline Enfermedades cardiovasculares (I00-I99) & 19,8 & 36,0 & 23,9 & 16,6 & 9,3 & 3,89 & \begin{tabular}{|l|l|}
1,98 & 7,65 \\
\end{tabular} & 26,7 & $12,6 \mid 40,8$ & 15,4 \\
\hline Suicidio (X60-X84) & 11,9 & 13,3 & 13,0 & 12,2 & 9,7 & 1,36 & $0,61 \mid 3,07$ & 3,5 & $-6,0 \mid 13,1$ & 2,0 \\
\hline \multicolumn{11}{|l|}{45 a 64} \\
\hline Todas las causas & 722,1 & 860,0 & 710,5 & 660,0 & 516,2 & 1,67 & \begin{tabular}{ll|l}
1,46 & 1,91 \\
\end{tabular} & 343,8 & $264,4 \mid 423,2$ & 100,0 \\
\hline Cáncer (C00-C99) & 360,0 & 408,1 & 359,6 & 347,1 & 283,5 & 1,44 & $1,20 \mid 1,73$ & 124,6 & $67,1 \mid 182,1$ & 36,2 \\
\hline Enfermedades cardiovasculares (I00-I99) & 152,7 & 172,9 & 150,5 & 149,9 & 116,2 & 1,49 & \begin{tabular}{l|l|l}
1,11 & 1,99 \\
\end{tabular} & 56,7 & $19,2 \mid 94,1$ & 16,5 \\
\hline Enfermedades digestivas (K00-K93) & 40,6 & 57,4 & 36,7 & 41,1 & 12,2 & 4,70 & \begin{tabular}{|l|l|}
2,02 & 10,94 \\
\end{tabular} & 45,2 & $29,1 \mid 61,2$ & 13,1 \\
\hline Accidentes no intencionales (W00-X59) & 39,7 & 58,8 & 39,2 & 23,2 & 16,4 & 3,57 & $1,80 \mid 7,11$ & 42,3 & $25,4 \mid 59,3$ & 12,3 \\
\hline \multicolumn{11}{|l|}{65 a 74} \\
\hline Todas las causas & $2.754,5$ & $2.887,3$ & $2.560,4$ & $2.711,4$ & $2.341,1$ & 1,23 & $1,19 \mid 1,28$ & 546,2 & $436,4 \mid 655,9$ & 100,0 \\
\hline Cáncer (C00-C99) & 1102,7 & $1.115,2$ & $1.094,9$ & $1.133,2$ & 983,2 & 1,13 & $1,07 \mid 1,21$ & 131,9 & $63,2 \mid 200,7$ & 24,1 \\
\hline Enfermedades cardiovasculares (I00-I99) & 736,5 & 779,2 & 657,0 & 739,6 & 682,5 & 1,14 & $1,06 \mid 1,23$ & 96,8 & \begin{tabular}{ll|l|}
39,6 & 156,9 \\
\end{tabular} & 17,7 \\
\hline Enfermedades respiratorias (J00-J99) & 284,7 & 330,6 & 206,1 & 184,5 & 257,0 & 1,29 & $1,15 \mid 1,44$ & 73,6 & \begin{tabular}{l|l|l}
36,8 & 110,4 \\
\end{tabular} & 13,5 \\
\hline Enfermedades digestivas & 128,5 & 144,0 & 116,5 & 113,8 & 48,2 & 2,99 & $2,48 \mid 3,59$ & 95,8 & $71,9 \mid 119,6$ & 17,5 \\
\hline \multicolumn{11}{|l|}{75 y más } \\
\hline Todas las causas & $11.181,4$ & $11.361,3$ & $10.971,6$ & $11.392,6$ & $9.353,0$ & 1,21 & $1,09 \mid 1,36$ & 2008,3 & $965,7 \mid 3.050,8$ & 100,0 \\
\hline Enfermedades cardiovasculares (I00-I99) & $3.646,0$ & $3.727,9$ & $3.527,2$ & $3.979,6$ & $2.746,4$ & 1,36 & $1,11 \mid 1,67$ & 981,5 & \begin{tabular}{|l|l|}
406,6 & $1.556,3$ \\
\end{tabular} & 48,9 \\
\hline Cáncer (C00-C99) & $2.293,3$ & $2.219,6$ & $2.500,0$ & $2.802,7$ & $2.187,1$ & 1,01 & $0,82 \mid 1,26$ & 32,5 & $-447,2 \mid 512,3$ & 1,6 \\
\hline Enfermedades respiratorias (J00-J99) & $1.998,1$ & $2.099,40$ & $1.744,10$ & $1.453,1$ & $1.749,2$ & 1,20 & $0,92 \mid 1,56$ & 350,2 & $-114,1 \mid 814,6$ & 17,4 \\
\hline Enfermedades digestivas (K00-K93) & 512,0 & 545,4 & 515,6 & 353,5 & 201,2 & 2,71 & $1,35 \mid 5,42$ & 344,1 & $192,3 \mid 496,0$ & 17,1 \\
\hline
\end{tabular}

${ }^{1} \mathrm{RT}=$ Razón entre la tasas de mortalidad en el grupo con educación primaria o menos y la tasa de mortalidad en el grupo de estudios universitarios. ${ }^{2}$ DT $=$ Diferencia entre la tasas de mortalidad en el grupo con educación primaria o menos y la tasa de mortalidad en el grupo de estudios universitarios. ${ }^{3}$ Representa la diferencia de tasas en esa causa de muerte con respecto a la diferencia en la tasa de mortalidad por todas las causas. 
Tabla 5

Tasa de mortalidad estandarizada por edad por 100.000 personas-año a riesgo

por las principales causas de muerte según el nivel educativo, razón de tasas de mortalidad (RT), diferencia de tasas de mortalidad por 100.000 personas-año (DT) e intervalos de confianza al 95\% (IC95\%) de la razón y la diferencia.

Cohorte de mujeres de diferentes grupos de edad en Navarra, 2001 a 2008

\begin{tabular}{|c|c|c|c|c|c|c|c|c|c|c|}
\hline Grupo de edad y causa de muerte & Total & $\begin{array}{c}\text { Educación } \\
\text { primaria } \\
\text { o menos }\end{array}$ & $\begin{array}{l}\text { Educación } \\
\text { secundaria } \\
\text { de } 1^{\circ} \text { ciclo }\end{array}$ & $\begin{array}{l}\text { Educación } \\
\text { secundaria } \\
\text { de } 2^{\circ} \text { ciclo }\end{array}$ & $\begin{array}{c}\text { Estudios } \\
\text { universitarios }\end{array}$ & $\mathrm{RT}^{1}$ & IC $95 \%$ & $\mathrm{DT}^{2}$ & IC $95 \%$ & $\begin{array}{c}\text { Contribución } \\
\text { a la diferencia } \\
\text { en la mortalidad } \\
\text { por todas } \\
\text { las causas }(\%)^{3}\end{array}$ \\
\hline
\end{tabular}

\section{5 a 44}

\begin{tabular}{|c|c|c|c|c|c|c|c|c|c|c|}
\hline Todas las causas & 67,3 & 116,5 & 81,6 & 64,0 & 42,8 & 2,72 & $1,95 \mid 3,80$ & 73,7 & $44,5 \mid 102,9$ & 100,0 \\
\hline Cáncer (C00-C99) & 32,5 & 33,2 & 38,7 & 38,5 & 21,5 & 1,54 & $0,90 \mid 2,65$ & 11,7 & \begin{tabular}{l|l|}
$-0,4$ & 27,4 \\
\end{tabular} & 15,9 \\
\hline Accidentes no intencionales (W00-X59) & 6,5 & 7,4 & 7,2 & 6,5 & 5,6 & 1,32 & $0.44 \mid 3.99$ & 1,8 & $-5.8 \mid 9.3$ & 2,4 \\
\hline Suicidio (X60-X84) & 5,1 & 7,7 & 4,9 & 6,4 & 3,9 & 1,95 & $0,55 \mid 6,89$ & 3,7 & $-0,4 \mid 12,0$ & 5,0 \\
\hline Enfermedades cardiovasculares (I00-I99) & 4,2 & 11,8 & 4,1 & 2,2 & 2,1 & 5,71 & $1,69 \mid 19,23$ & 9,8 & $0,2 \mid 17,8$ & 13,3 \\
\hline \multicolumn{11}{|l|}{45 a 64} \\
\hline Todas las causas & 245,1 & 268,4 & 224,0 & 245,4 & 258,9 & 1,04 & $0,84 \mid 1,28$ & 9,5 & $-46,5 \mid 65,5$ & 100,0 \\
\hline Cáncer (C00-C99) & 171,0 & 177,4 & 155,0 & 180,2 & 204,4 & 0,87 & $0,68 \mid 1,11$ & $-27,0$ & $-76,6 \mid 22,7$ & $-284,2$ \\
\hline Enfermedades cardiovasculares (I00-I99) & 41,6 & 55,4 & 32,1 & 40,6 & 19,0 & 2,92 & $1,41 \mid 6,04$ & 36,4 & $18,8 \mid 54,0$ & 383,2 \\
\hline Enfermedades respiratorias (J00-J99) & 12,9 & 16,7 & 8,5 & 7,1 & 12,0 & 1,39 & $0,48 \mid 4,07$ & 4,7 & $-0,9 \mid 18,2$ & 49,5 \\
\hline Accidentes no intencionales (W00-X59) & 12,1 & 13,7 & 11,3 & 10,0 & 7,6 & 1,81 & $0,67 \mid 4,86$ & 6,1 & $-2,7 \mid 15,1$ & 64,2 \\
\hline \multicolumn{11}{|l|}{65 a 74} \\
\hline Todas las causas & $1.209,6$ & $1.279,0$ & $1.097,1$ & 922,6 & $1.056,6$ & 1,21 & $0,96 \mid 1,54$ & 222,3 & $-24,7 \mid 493,6$ & 100,0 \\
\hline Cáncer (C00-C99) & 438,7 & 440,8 & 447,3 & 350,0 & 463,4 & 0,95 & $0,67 \mid 1,35$ & $-22,7$ & $-185,0 \mid 140,0$ & $-10,2$ \\
\hline Enfermedades cardiovasculares (I00-I99) & 327,1 & 358,3 & 277,4 & 208,9 & 225,5 & 1,59 & $0,97 \mid 2,62$ & 132,8 & \begin{tabular}{ll|l|}
17,6 & 248,0 \\
\end{tabular} & 59,7 \\
\hline Enfermedades respiratorias (J00-J99) & 91,5 & 99,4 & 82,0 & 35,1 & 71,1 & 1,40 & $0,57 \mid 42$ & 28,3 & $-36,2 \mid 92,9$ & 12,7 \\
\hline Enfermedades digestivas (K00-K93) & 54,1 & 53,9 & 53,4 & 94,6 & 13,8 & 3,90 & \begin{tabular}{l|l|l|}
0,54 & 28,06 \\
\end{tabular} & 40,0 & $10,1 \mid 70,0$ & 18,0 \\
\hline \multicolumn{11}{|l|}{75 y más } \\
\hline Todas las causas & $7.317,9$ & $7.532,8$ & $6.678,2$ & $6.480,8$ & $6.223,9$ & 1,21 & $1,06 \quad 1,38$ & 1308,9 & $\begin{array}{l}486,4 \mid 2131,3 \\
\end{array}$ & 100,0 \\
\hline Enfermedades cardiovasculares (I00-I99) & $2.742,2$ & $2.821,8$ & $2.537,9$ & $2.405,1$ & $2.257,2$ & 1,25 & $1,00 \quad 1,56$ & 564,6 & $67,8 \mid 1061,3$ & 43,1 \\
\hline Cáncer (C00-C99) & 969,7 & 955,1 & 974,9 & $1.229,7$ & $1.088,3$ & 0,88 & $0,64 \quad 1,20$ & $-133,2$ & $-471,9 \mid 205,6$ & $-10,2$ \\
\hline Enfermedades respiratorias (J00-J99) & 892,5 & 947,8 & 729,1 & 726,9 & 463,3 & 2,05 & $1,273,31$ & 484,5 & $256,3 \mid 712,7$ & 37,0 \\
\hline Enfermedades digestivas (K00-K93) & 363,2 & 383,9 & 287,7 & 284,7 & 369,3 & 1,04 & $0,61 \quad 1,77$ & 14,6 & $-182,5 \mid 211,7$ & 1,1 \\
\hline
\end{tabular}

${ }^{1} \mathrm{RT}=$ Razón entre la tasas de mortalidad en el grupo con educación primaria o menos y la tasa de mortalidad en el grupo de estudios universitarios. ${ }^{2} \mathrm{DT}=\mathrm{Diferencia}$ entre la tsas de mortalidad en el grupo con educación primaria o menos y la tasa de mortalidad en el grupo de estudios universitarios. ${ }^{3}$ Representa la diferencia de tasas en esa causa de muerte con respecto a la diferencia en la tasa de mortalidad por todas las causas. 


\section{DISCUSIÓN}

La tasa de mortalidad en la población navarra muestra un gradiente inverso con el nivel educativo. Este gradiente se observa para las principales causas de muerte, a excepción de algunas localizaciones de cáncer. El VIH es la causa de muerte que presenta una mayor razón de tasas de mortalidad entre las personas con menor y mayor nivel de estudios. Otras causas de muerte con elevada razón de tasas de mortalidad entre ambas categorías de nivel de estudios son los accidentes y las enfermedades del aparato digestivo en hombres y la diabetes mellitus en mujeres. Las causas de muerte que contribuyen en mayor proporción a la diferencia absoluta entre las tasa de mortalidad en las categorías baja y alta de nivel de estudios son las enfermedades cardiovasculares, el cáncer y las enfermedades del aparato digestivo en hombres y las enfermedades cardiovasculares, enfermedades respiratorias y la diabetes mellitus en mujeres. En los diferentes grupos de edad, también se observa un gradiente inverso de la tasa de mortalidad, a excepción de la mortalidad por cáncer en las mujeres mayores de 45 años.

Las diferencias relativas en la tasa de mortalidad total según la educación, estimadas mediante la razón de tasas de mortalidad, presentan menor magnitud que las diferencias obtenidas en otras investigaciones realizadas en poblaciones de Italia $^{4}$, Canadá ${ }^{5}$, Estonia ${ }^{6}$, Lituania $^{7}$, Suiza $^{8}$ o los países nórdicos ${ }^{9}$. Así mismo, esas diferencias en mujeres son menores que las obtenidas para el conjunto de mujeres españolas ${ }^{10}$. Ese hecho se debe a que la razón de tasas de mortalidad por cáncer en las mujeres españolas está por encima de la unidad, mientras que en las mujeres navarras es de 0,92 . Por otro lado, al igual que los resultados obtenidos en la presente investigación, la mayoría de los estudios sobre las diferencias relativas en mortalidad según el nivel de estudios en los países desarrollados, encuentran que la magnitud es mayor en los hombres que en las mujeres ${ }^{11}$. Dos estudios ecológicos realizados en Navarra y en la ciu- dad de Pamplona, en los que se compararon las tasas de mortalidad según la privación económica del área de residencia, también encontraron mayor magnitud en hombres que en mujeres de las diferencias socioeconómicas relativas en mortalidad ${ }^{12,13}$.

El VIH es la causa de muerte que muestra la razón de tasas de mortalidad más alta, sobre todo en personas de 25 a 44 años (datos no mostrados). Esto se debe a la epidemia de uso de drogas por vía parenteral en España, que alcanzó su punto máximo a finales de los 80 y los principales afectados fueron los adultos jóvenes de menor posición socioeconómica ${ }^{14}$. Sin embargo, debido a que la tasa de mortalidad por sida es muy baja en comparación con otras causas de muerte, su contribución a la diferencia absoluta de mortalidad entre las personas con menor y mayor nivel de estudios es insignificante.

En cambio, las enfermedades cardiovasculares, que presentan una razón de tasas de mortalidad muy baja, constituyen la causa de muerte que más contribuye a la diferencia absoluta en mortalidad entre las personas con mayor y menor nivel de estudios: un 26\% en hombres y un $48 \%$ en mujeres. La menor proporción de muertes por enfermedad cardiovascular en los hombres en comparación con las mujeres y el hecho de que la razón de tasas de mortalidad por esta causa de muerte también sea menor en los hombres, explica por qué la contribución de la enfermedad cardiovascular es mayor en las mujeres.

Casi un 20\% de la diferencia absoluta en la tasa de mortalidad en hombres es debida al cáncer. En cambio, en mujeres la contribución es negativa. Este hecho se debe a que en las mujeres navarras mayores de 45 años la mortalidad por cáncer es mayor en las que tienen educación universitaria. Se trata de un hallazgo similar al obtenido en algunas regiones españolas ${ }^{15}$. Probablemente este hecho se deba a que las mujeres españolas con mayor nivel de estudios presenten mayor prevalencia de algunos factores de riesgo relacionados con algunas localizaciones de cáncer, ta- 
les como un retraso en la edad gestacional, una menor duración de la lactancia materna o una mayor frecuencia de fumar ${ }^{16-18}$. En contraste, en los hombres, la mortalidad por cáncer muestra un gradiente inverso con el nivel de estudios. En algunas localizaciones de cáncer este hallazgo podría atribuirse a la mayor frecuencia de tabaquismo en hombres con un nivel de estudios bajo ${ }^{18}$.

En mujeres, las enfermedades del aparato respiratorio constituyen la segunda causa de muerte que contribuye a la diferencia absoluta en las tasas de mortalidad. Este hallazgo también se ha observado en otras regiones españolas ${ }^{15}$. Se trata de un resultado sorprendente de difícil explicación, puesto que el tabaquismo, uno de los principales factores de riesgo de estas enfermedades, es más frecuente en las mujeres españolas de nivel de estudios altos ${ }^{19}$, mientras que la razón de tasas de mortalidad en mujeres con menor y mayor nivel de estudios es 1,87. En el futuro habrá que investigar posibles factores que podrían explicar este hallazgo, como, por ejemplo, una posible diferencia socioeconómica en el pronóstico de estas enfermedades.

En hombres, las enfermedades del aparato digestivo constituyen la tercera causa de muerte que contribuye a la diferencia absoluta en las tasas de mortalidad. Este hallazgo se debe a la elevada razón de tasas de mortalidad por esta causa entre los sujetos con menor y mayor nivel de estudios. El gradiente inverso con el nivel de estudios de la prevalencia de consumo excesivo de alcohol en los hombres puede ser responsable de este hallazgo, ya que es un factor de riesgo asociado con enfermedades digestivas, tales como úlcera péptica, pancreatitis y cirrosis hepática $^{20}$. Otra causa de muerte con elevada razón de tasas de mortalidad, como los accidentes no intencionales, contribuye en menor proporción a las diferencias absolutas en mortalidad, debido a que la tasa de mortalidad no es muy elevada. En cambio, en el grupo de 25 a 44 años, donde constitu- ye la primera causa de muerte, es responsable de un $30 \%$ de la diferencia absoluta en mortalidad.

En el caso de la diabetes mellitus, la elevada razón de tasas de mortalidad es responsable de que esta causa de muerte sea las tercera que más contribuye a la diferencia absoluta en las tasas de mortalidad en mujeres. Se trata de un hallazgo que también se ha observado en el conjunto de las mujeres españolas ${ }^{10}$ y que puede atribuirse fundamentalmente al gradiente inverso en la prevalencia de obesidad según el nivel de estudios ${ }^{21}$. Si se tiene en cuenta que las enfermedades cardiovasculares comparten factores de riesgo con la diabetes mellitus, puede señalarse que el $60 \%$ de la diferencia absoluta entre las tasas de mortalidad en las mujeres con menor y mayor nivel de estudios podrían ser explicadas por las diferencias socioeconómicas en la prevalencia de algunos factores de riesgo cardiovascular como la obesidad ${ }^{21}$ o la inactividad física ${ }^{22}$.

Este es el primer estudio en Navarra para obtener estimaciones regionales de las diferencias de mortalidad por nivel educativo en toda la población. Es un estudio sin problemas de representatividad, ya que incluye a todas las personas de 25 años o más años que residían en Navarra en el momento del censo de población de 2001. Por otro lado, además de las diferencias relativas se presentan las diferencias absolutas, lo que permite identificar las causas de muerte que contribuyen en mayor proporción a las diferencias en mortalidad total entre los sujetos con mayor y menor educación.

El nivel educativo se considera como una medida de posición socioeconómica que está directamente relacionado con la salud a través de su correlación con las condiciones socioeconómicas, factores psicológicos y estilo de vida. Es fácil de interpretar y constituye una medida sencilla para la clasificación de toda la población y no solo de los que tienen una ocupación ${ }^{23}$. 
Sin embargo, este estudio está basado en la información de sujetos que pertenecen a varias cohortes de nacimiento. Como consecuencia, hay gran heterogeneidad en la distribución del nivel de estudios según la edad. Por ejemplo, solo el 10\% de las mujeres de 25 a 44 años pertenecen a la categoría más baja de nivel de estudios, mientras que este porcentaje asciende al 76,6\% en las mujeres de 75 y más años. Por tanto, es posible que el significado de la educación no sea el mismo en la población joven que en la población de mayor edad ${ }^{24}$. Dado el mayor acceso a la educación en la población joven, un nivel de estudios bajo en los adultos jóvenes refleja unas condiciones de vida más adversas que en la población de mayor edad. No obstante, las estimaciones de las diferencias en mortalidad según la educación por grupos de edad muestran un patrón similar a la que se observa con las estimaciones en el conjunto de la población.

Las diferencias en mortalidad por diversas causas de muerte podrían estar relacionadas con el patrón socioeconómico en la prevalencia de factores de riesgo observado en estudios previos. La ausencia de esos factores de riesgo en los datos analizados impide conocer hasta qué punto la distribución desigual de los factores explica los hallazgos obtenidos.

En resumen, los hallazgos de la presente investigación aportan abundante evidencia empírica que puede incorporarse a la agenda política del Plan de Salud de Navarra 20142020. Por ejemplo, a la hora de establecer prioridades de intervención, los responsables deberán establecer los objetivos concretos a lograr. Mientras que algunas causas de muerte con elevadas diferencias relativas en mortalidad contribuyen muy poco a las diferencias absolutas en mortalidad, otras causas de muerte con bajas diferencias relativas en mortalidad representan una carga importante de enfermedad y contribuyen en gran medida a las diferencias absolutas en las tasas de mortalidad.

\section{AGRADECIMIENTOS}

Este estudio ha sido realizado dentro del Proyecto "Desigualdades socioeconómicas en la mortalidad total y en la mortalidad por causa de muerte en Navarra", que fue financiado por la Fundación Caja Navarra.

\section{BIBLIOGRAFÍA}

1. Borrell C, Peiró R, Ramón N, Pasarín MI, Colomer C, Zafra E, Alvarez-Dardet C. Desigualdades socioeconómicas y planes de salud en las comunidades autónomas del Estado español. Gac Sanit. 2005;19(4):277-85.

2. Plan de Salud de Navarra 2014-2020. https://www. navarra.es/NR/rdonlyres/E0566B9B-2D62-423D9EA6-72DB748E390A/303761/PLANDESALUD20142020versionfinalParlamento.pdf

3. Greenland S, Rothman KJ. Introduction to stratified analysis. In: Rothman KJ, Greenland S (eds). Modern Epidemiology. Philadelphia: Lippincott Williamsn \& Wilkins; 1998:253-280.

4. Marinacci C, Grippo F, Pappagallo M, Sebastiani G, Demaria M, Vittori P, Caranci N, Costa G. Social inequalities in total and cause-specific mortality of a sample of the Italian population, from 1999 to 2007. Eur J Public Health. 2013;23:582-7.

5. Tjepkema M, Wilkins R, Long A. Cause-specific mortality by education in Canada: a 16-year follow-up study. Health Rep. 2012;23:23-31.

6. Leinsalu M, Vågerö D, Kunst AE. Estonia 1989-2000: enormous increase in mortality differences by education. Int J Epidemiol. 2003;32:1081-7.

7. Kalediene R, Petrauskiene J. Inequalities in mortality by education and socio-economic transition in Lithuania: equal opportunities? Public Health. 2005;119:808-15.

8. Bopp M, Minder CE, Swiss National Cohort. Mortality by education in German speaking Switzerland, 19901997: results from the Swiss National Cohort. Int J Epidemiol. 2003;32:346-54.

9. Shkolnikov VM, Andreev EM, Jdanov DA, Jasilionis D, Kravdal Ø, Vågerö D, Valkonen T. Increasing absolute mortality disparities by education in Finland, Norway and Sweden, 1971-2000. J Epidemiol Community Health. 2012; 66:372-8.

10. Reques L, Giráldez-García C, Miqueleiz E, Belza MJ, Regidor E. Educational differences in mortality and the relative importance of different causes of death: a 7-year follow-up study of Spanish adults. J Epidemiol Community Health. 2014;68(12):1151-60. 
11. Regidor E, Calle ME, Navarro P, Domínguez V. The size of educational differences in mortality from specific causes of death in men and women. Eur J Epidemiol. 2003;18(5):395-400.

12. Casi Casanellas A, Moreno Iribas C. Desigualdad ante la muerte: Estudio comparativo entre comunidades de Navarra en el segmento de población de 25 a 74 años. Aten Primaria. 1992; 10: 543-548.

13. Izco Goñi N, Etxeberria Andueza J, Delfrade Osinaga J, Floristán Floristán Y, Moreno Iribas C. y otros miembros de MEDEA. Desigualdades socioeconómicas en la mortalidad en Pamplona y Logroño en el periodo 1996-2007. An Sist Sanit Navar. 2013; 36,2.

14. Regidor E, De la Fuente L, Calle ME, et al. Unusually strong association between education and mortality in young adults in a community with a high rate of injection-drug users. Eur J Public Health. 2003;13:334-9.

15. Mackenbach JP, Stirbu I, Roskam AJ, et al. Socioeconomic inequalities in health in 22 European countries. N Engl J Med. 2008;358:2468-81.

16. Strand BH, Kunst A, Huisman M, et al. The reversed social gradient: higher breast cancer mortality in the higher educated compared to lower educated. A comparison of 11 European populations during the 1990s. Eur J Cancer. 2007;43:1200-7.

17. Merletti F, Galassi C, Spadea T. The socioeconomic determinants of cancer. Environ Health 2011;10(Suppl 1):S7.

18. Huisman M, Kunst AE, Mackenbach JP. Educational inequalities in smoking among men and women aged 16 years and older in 11 European countries. Tob Control. 2005;14:106-13.

19. Bacigalupe A, Esnaola S, Martín U, Borrell C. Two decades of inequalities in smoking prevalence, initiation and cessation in a southern European region: 1986-2007. Eur J Public Health. 2013 Aug;23(4):5528.

20. Corrao G, Bagnardi V, Zambon A, La Vecchia C. A meta-analysis of alcohol consumption and the risk of 15 diseases. Prev Med. 2004;38:613-19.

21. Gutiérrez-Fisac JL, Guallar-Castillón P, León-Muñoz LM, et al. Prevalence of general and abdominal obesity in the adult population of Spain, 2008-2010: the ENRICA study. Obes Rev. 2012;13:388-92.

22. Maestre-Miquel C, Martínez D, Polonio B, Astasio P, Santos J, Regidor E. Desigualdades en inactividad física según el nivel de estudios en España, en 1987 y 2007. Aten Primaria. 2014;46(10):565-72.
23. Ross CE, Mirowsky J. Refining the association between education and health: the effects of quantity, credential, and selectivity. Demography. 1999;36:445-60.

24. Hadden WC. Annotation: the use of educational attainment as an indicator of socioeconomic position. Am J Public Health. 1996;86(11):1525-6. 